\title{
Joint Source-Channel Coding and Digital Modulation Technique with Index Assignment Optimization
}

\author{
Tran Ngoc Tuan, Nguyen Quoc Trung \\ School of Electronics and Telecommunications, Hanoi University of Science and Technology \\ Email: tuan.tranngoc@hust.edu.vn, trung.nguyenquoc@hust.edu.vn
}

\begin{abstract}
Channel optimized Index Assignment (IA) of source codeword is an approach to improve the transmission error resilience without the addition of extra bits and coding/decoding complexity. In this paper, we study the IA scheme with consideration to the given modulation technique. This study also presents an IA scheme for joint source channel coding and modulation technique for the general case when the codeword is not required to correspond to a modulation symbol and to occupy consecutive bits of the transmitted bitstream as some prior works. Besides the improvement in channel error robustness for the traditional IA approach, the proposed scheme can be combined with other techniques of mitigating or controlling the channel error when needed (in systems with high reliability or transmission of important codeword) such as Cyclic Redundancy Check (CRC), Forward Error Correction (FEC) coding and interleaving. We also consider the combination of the IA approach and FEC coding and the effectiveness of the proposed scheme is confirmed by experiments.
\end{abstract}

Keywords - Joint Source-Channel Coding, Index Assignment, Vector Quantization, Source-Channel coding and Modulation ...

\section{INTRODUCTION}

When transmitting analog source signals over the communication system, the most common approach is to use separate source and channel coders. This strategy, known as the "separation principle", was introduced and shown to be optimum by Shannon [1]. However, this theorem is true given infinite complexity and delay in the coders, which is seldom met in practical situations. Thus, it is advantageous to design the source and channel codes jointly, as witnessed by a large body of literature on joint source channel coding (JSCC). For a review of various JSCC techniques, see [5].

This paper is mainly concerned with a JSCC approach called optimization of Index Assignment (IA) applied to communication system transmitting continuous amplitude sources. The advantage of IA approach over others JSCC approach is that it does not require the channel known nor does it add any redundant bits to the bit stream and any complexity to the encoder/decoder

In this JSCC approach, the quantizer in the source coder, either scalar or vector, is optimized for the channel conditions. Systems based on Vector Quantization (VQ) is considered here since VQ is the general case of scalar quantization and is an effective and widely-used method for low-bit-rate coding of correlation signals such as speech, audio, image and video [2].

Most previous works on this approach are based on assuming a binary symmetric channel (BSC) and forcus on AWGN channel, regardless of the modulation type. However, depending on the modulation type and the channel model, such assumption is only valid for some particular cases (binary modulation or low channel noise).

There are also some JSCC methods reported in the literature with regard to the modulation type. In $[9,11]$, the constellation diagram is modified in an optimal way according to the source and channel charracteristic and the system need to be redesigned using modulation with uncommon constellation diagram. Moreover, these methods cannot be simultaneously utilized to optimize the transmission of various codewords in frame. Some other methods only considers the special case $[7,10,12]$ when a codeword is corresponding to a modulation symbol, but this is not suitable for many practical systems.

Recently, the study in [8] proposed an IA method with regard to the given modulation types for the case that the modulation is two-dimensional (2D) signaling multilevel and the codeword is not necessary to be fully allocated into a whole number of modulation 
Volume E-3 No. 9 (13)

symbols. This method preserves the advantages of tradditional IA method as mentioned above, while giving better performance than IA methods without considering the modulation type. However, this method is constrained by the assumption that the codeword occupies consecutive bits of the transmitted bitstream, so it cannot be combined with any bitstream processing method for error control or mitigation (e.g. FEC, CRC or interleaving...). This method is not suitable to utilize in system requiring high reliability as well as to upgrade existing system using bitstream processing method.

In the present work, we extend the study in [8] and developed an IA method with consideration of the given modulation type, applied to systems transmitting continuous amplitude sources over discrete memoryless channel (DMC). The case considered here is not bound by the assumption as in [8] (the codeword occupies consecutive bits of the transmitted bitstream). As a result, in addition to improving the transmission robustness for more complicated case, the novel method can be combined with other error control or mitigation techniques and also be utilized to upgrade the existing systems using such techniques. Moreover, the transmission of multiple codewords per frame can be simultaneously optimized by applying this IA scheme to each codeword in turn.

Subsequently, we summarize an IA scheme for joint source-channel coding and modulation type and consider the combination of IA approach and FEC coding. The effectiveness of the proposed method is evaluated by simulations.

The remainder of the paper is structured as follows. Section 2 formulates the problem of optimal Index Assignment. In section 3, we discuss the combined source channel coding and digital modulation types approach and present a new IA method with consideration of the given modulation technique. The performance of the proposed IA method is envaluated by simulations in Section 4. And finally, some conclusions and directions for future work are given in Section 5.

\section{PROBLEM ForMulation}

\subsection{Signal Quantization.}

The basic element of digital communication systems is the quantizer, either scalar or vector. In this paper, we consider vector quantization because this is the general case of scalar quantization.

When a set of discrete-time amplitude values is quantized jointly as a single vector, the process is known as Vector Quantization (VQ) [2], also known as block quantization or pattern matching quantization. A vector quantizer $Q: \mathfrak{R}^{K} \rightarrow \mathrm{C}$ maps a continuous source vector $\boldsymbol{x} \in \mathfrak{R}^{K}$ to a codevector $c_{i} \in \mathbf{C}$ by the nearest neighbour rule. The $n$-bit codebook $\mathbf{C}=\left\{\boldsymbol{c}_{\boldsymbol{i}} ; 0 \leq i \leq N-1\right\}$ is the set of $K$ dimensional codevectors.

The principle of VQ is shown in Fig.1. With a given code book $\mathbf{C}$, the vector quantization task is to replace an input vector $\boldsymbol{x}$ by the most similar vector $c_{i}$. The choice is based on a distance or distortion measure $d\left(x, c_{i}\right)$, and a widely used distance measure is the squared Euclidean distance, given by:

$$
d\left(x, c_{i}\right)=\left\|x-c_{i}\right\|^{2}
$$

The output of the encoder is the index $i$ of the codevector $c_{i}$ which is the satisfies:

$$
i=\underset{k}{\operatorname{argmin}} d\left(x, c_{k}\right)
$$

Only the index $i$ is transmitted over the channel to the receiver. Upon receiving $i$ correctly, the VQ decoder can reconstruct $\boldsymbol{x}$ to $\boldsymbol{c}_{\boldsymbol{i}}$ by a simple table lookup operation.

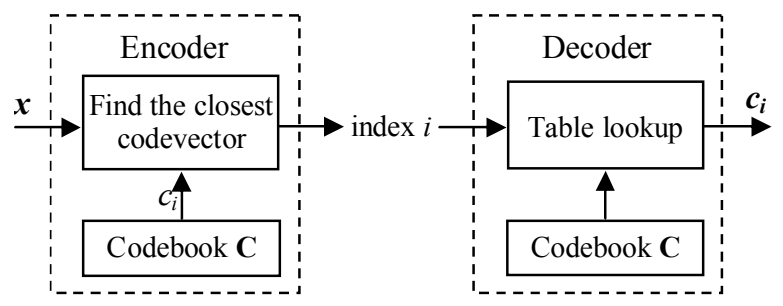

Fig. 1 Principle of vector quantization

The design principle of a VQ consists of partitioning the $K$-dimensional space of source vectors $\boldsymbol{x}$ into $N$ non overlapping cells $\left\{\mathrm{R}_{1}, \mathrm{R}_{2}, \ldots \mathrm{R}_{\mathrm{N}}\right\}$ and associating with each cell $\mathrm{R}_{i}$ a unique codevector 
(centroid) $\boldsymbol{c}_{\boldsymbol{i}}$ such as the total average distortion is minimized. A well-known algorithm for VQ codebook design is the Linde-Buzo-Gray (LBG) algorithm [4] (refer to [6] for the survey of VQ codebook generation algorithms).

\subsection{Index Assignment.}

On a noiseless channel, the IA of the codevectors does not affect the system performance. However, in the absence of channel noise, the received indices may have errors which results in significant distortion in decoded vectors, so the IA plays an important role in determining the overall system performance.

Let $P_{a}(i)$ denote the a priori probability of codevector $\boldsymbol{c}_{\boldsymbol{i}} \in \mathrm{C}$ and $P_{\mathrm{C}}(i, j)$ be the probability of transmitting index $j$ but receiving index $i$. Any assignment of $n$-bit indices to codevectors in $\mathbf{C}$ is obtained by a permutation $\pi$ of the integers $\{0,1, \ldots, N-1\}$ and association of index $\pi(i)$ to codevector $\boldsymbol{c}_{\boldsymbol{i}}$. The sum of possible distortions when transmitting vector $\boldsymbol{c}_{\mathbf{i}}$ is:

$$
D_{i}(\pi)=P_{a}(i) \sum_{j=1}^{N} P_{C}(\pi(j), \pi(i)) d(i, j)
$$

The overall distortion caused by channel noise is:

$$
D(\pi)=\sum_{i=1}^{N} P_{a}(i) \sum_{j=1}^{N} P_{C}(\pi(j), \pi(i)) d(i, j)
$$

Different index assignments do not change the distortion of the source code, but they do affect the overall distortion of a communication system $D(\pi)$ in case of channel error. The IA problem is to find the best codebook rearrangement $\pi$ which minimize $D(\pi)$ for given input parameters $P_{a}(i), P_{C}(i, j)$ and $d(i, j)$. There are $N$ ! possibilities to order $N$ codewords, and to find an optimal solution for codebooks larger than 32 entries is practically impossible. For this reason, a number of different suboptimal IA approachs have been proposed [13-19].

In addition, in case of using no IA optimization method, there is a widely-used method, an option of the LBG algorithm, that is to use spliting procedure to generate the initial codebook for LBG algorithm [4]. In this way, the result codebook has a natural ordering that can somehow mitigate the signal distortion caused by channel error [14].

\section{COMBINED SOUCE-CHANNEL CODING AND DIGITAL MODULATION TYPES.}

To implement IA methods, the input parameter $P_{a}(i), P_{C}(i, j)$ and $d(i, j)$ need to be calculated first. The a priori probabilities of the codevector $P_{a}(i)$ can be estimated from the training set and the parameters $d(i, j)$ can be calculated from the codebook C. The remaining parameter $P_{C}(i, j)$ depend on the modulation type and the channel model. If we solve the IA problem regardless of modulation technique, the parameters $P_{C}(a, b)$ is calculated with the assumption of BSC channel as follows:

$$
P_{\mathbf{C}}(i, j)=\varepsilon^{h(i, j)}(1-\varepsilon)^{n-h(i, j)}
$$

where $\varepsilon$ is the bit error probability and $h(i, j)$ denote the Hamming distance (number of bit differences) between $i$ and $j$.

This assumption is only valid when the modulation scheme is binary or QPSK with Gray coding, but in the case of multilevel modulation, the above calculation of $P_{\mathrm{C}}(i, j)$ is only approximate in nature. On the other hand, if the modulation type is taken into account, the parameters $P_{C}(i, j)$ will be calculated more exactly and the performance of the system can be improved.

As mentioned in Section 1, in this work, the IA problem is extended to the more general case, where the codeword is not required to occupy consecutive bits of the transmitted bitstream and the transmission channel is DMC. The DMC, whose special case is $\mathrm{BSC}$, is a common model of channel and is appropriate for studying digital communication systems transmitting over memoryless channel. On the other hand, in the case of channel with memory, the channel model can be converted to DMC by scrambling techniques. The system model is described in detail in Section 3.1.

In case of binary modulation, the codeword transition probability parameters can be calculated using Eq.(5), so this study focuses on the case of multilevel modulation. After developing a method for evaluating these parameters in Section 3.2, we 
summarize cases relating to various modulation schemes and propose an IA scheme of joint sourcechannel coding and modulation type in Section 3.3.

\subsection{System model.}

We consider a communication system transmitting continuous amplitude sources and using VQ-based source coding. The source coder decomposes the input signal into a set of parameters. The fixed-length $n$-bit codeword being considered is multiplexed into a frame together with other codewords and bits. Let $l$ and $q$ be the length of the frame and the position of the codeword in the frame. The frames are modulated by a $M$-ary modulator in which their bits are mapped into $m$-bit symbols before modulated and transmitted over the physical channel. Assuming that the channel is memoryless, the combination of $M$-ary input in the modulator, the channel and the $M$-ary output in the demodulator forms a DMC. The block diagram of the system model is shown in Fig.2.

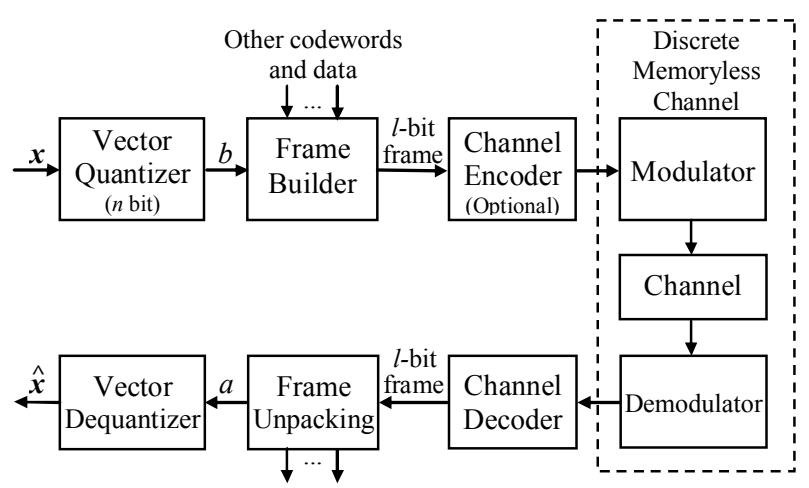

Fig. 2. Block diagram of the system model

An error control or mitigation method may be applied to increase the reliability of the transmission. The methods which can be applied here are bitstream process methods, including redundant bits insertion and bitstream rearrangement with the assumption of no change in the bits of the original data frame. The FEC code can be used in this model (if any) is systematic code in which the encoding process does not alter the original block of data bits and the parity bits are added as a separate part of the block.

Fig.3a depicts the case that the codeword $n$ bit is allocated into successive $m$-bit symbols so the codeword always occupies consecutive bits of symbol (no use of bit stream processing technique). Fig.3b describes an example in which codeword $n$ bit does not occupies consecutive bits of $m$-bit symbol due to the use of $\left(n_{b}, k_{b}\right)$ systematic FEC code (append $n_{b}-k_{b}$ parity bits to the beginning or end of each block of $k_{b}$ data bits, making each block $n_{b}$ bits long [20]).

It can be seen that the codeword transition probability depends on the symbol transition probabilities $P_{s}\left(s_{i}, s_{j}\right)$, the probability of transmitting symbol $s_{j}$ but receiving symbol $s_{i}\left(0 \leq s_{i}, s_{j} \leq M-1\right)$ :

$$
P_{\mathrm{S}}\left(s_{\mathrm{i}}, s_{\mathrm{j}}\right) \triangleq P\left(s_{i} \mid s_{\mathrm{j}}\right)
$$

The symbol transition probability for transmittion over AWGN channels and fading channels with arbitrary 2-D signaling can be exactly calculated by the signal-space partitioning method proposed in [3].

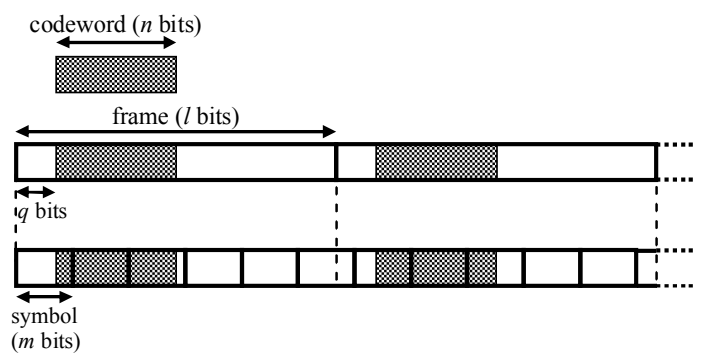

\section{(a) system using no error control or mitigation technique}

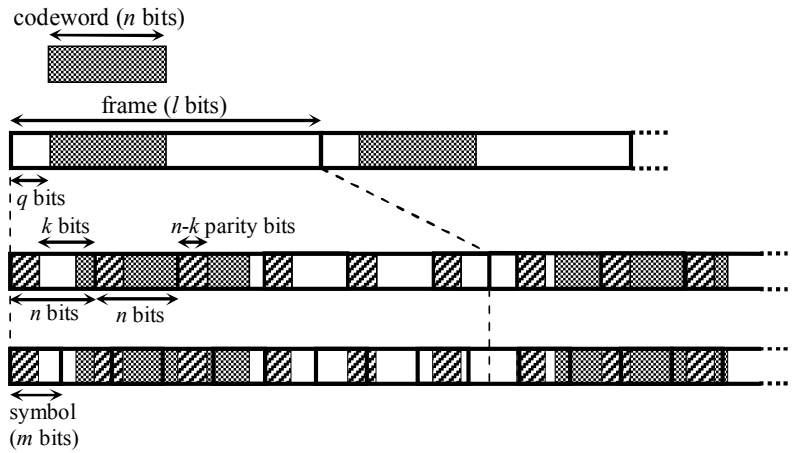

(b) system using $\left(n_{b}, k_{b}\right)$ systematic code

Fig. 3. An example of the allocation process of the $n$-bit codeword $b_{1} b_{2} \ldots b_{n}$ into m-bit symbols

In case the system is too complicated and it is impossible to apply this system model (e.g. using other channel models or combined with nonsystematic FEC codes), the IA method regardless of modulation type (with approximate input parameters estimation) can still be applied. 
3.2. Calculation of the parameters $P_{C}(a, b)$ in the case of multilevel modulation.

Assuming the system model introduced in Section 3.1, the purpose of this Section is to calculate the codeword transition probability $P_{\mathrm{C}}(a, b)$, the probability of transmitting codeword $b$ but receiving codeword $a$.

Firstly, it is necessary to determine all $p_{n}$ possibilities for allocating the $n$-bit codeword $b$ into symbols of length $m$ bits. For the $k$-th allocation possibility, the codeword $b$ is allocated into $n_{s k}$ symbols $s_{k, 1}^{(b)}, s_{k, 2}^{(b)}, \ldots, s_{k, n_{k}}^{(b)}$, and likewise, the codeword $a$ is also represented by $n_{\mathrm{sk}}$ symbols $s_{k, 1}^{(a)}, s_{k, 2}^{(a)}, \ldots, s_{k, n_{k+}}^{(a)}$.

These symbols may include $x$-bits which are bits in the frame but not belong to the codeword under consideration. At the transmitter, these $x$-bits are categorized as two types: determined bits $x_{d}$ whose values are fixed and undetermined bits $x_{u}$. At the receiver, all $x$-bits of codeword $a$ are undetermined. Let $n_{k, i}^{\left(x_{u}\right)}$ and $n_{k, i}^{\left(x_{d}\right)}$ denotes the number of $x_{u}$-bits and $x_{d^{-}}$-bits in $s_{k, i}^{(b)}$ respectively, therefore, the number of $x_{\mathrm{u}^{-}}$ bits in $s_{k, i}^{(a)}$ is $n_{k, i}^{\left(x_{i}\right)}+n_{k, i}^{\left(x_{d}\right)}$.

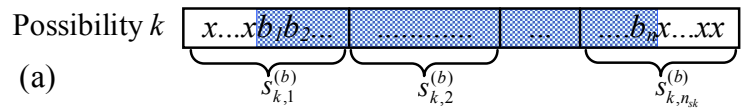

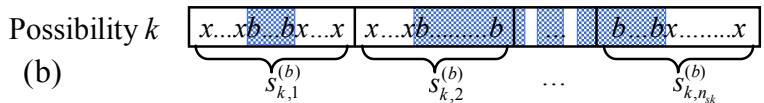

Fig. 4. A possibility for allocating the $n$-bit codeword $b$ into m-bit symbols; (a) system without using any error control or mitigation technique; (b) system using error control or mitigation technique

Fig.4 illustrates the $k$-th possibility of allocation of the codeword $b$ into $m$-bit symbols $s_{k, 1}^{(b)}, s_{k, 2}^{(b)}, \ldots, s_{k, n_{s k}}^{(b)}$ with two cases. One is the case of system without using any error control or mitigation technique (Fig.4a), the codeword $b$ is allocated into continuous symbols and occupies consecutive bits in the bitstream. The maximum number of allocation possibilities in this case is $m$ [8]. The other is the case that the codeword $b$ does not occupy consecutive bits of the transmitted bitstream (Fig.4b) due to the use of a bitstream process method for error control or mitigation. In case the number of allocation possibilities is large, all such possibilities can be explored by computer.

Secondly, evaluating the codeword transition probability for each allocation possibility. The probability corresponding to the $k$-th allocation possibility $P_{C_{k}}(a, b)$ for discrete memoryless channel (DMC) is the product of $n_{s k}$ probabilities:

$$
P_{C_{k}}(a, b)=\prod_{i=1}^{n_{s k}} P\left(s_{k, i}^{(a)} \mid s_{k, i}^{(b)}\right)
$$

where $P\left(s_{k, i}^{(a)} \mid s_{k, i}^{(b)}\right)$ is the probability of transmitting symbol $s_{k, i}^{(b)}$ but receiving symbol $s_{k, i}^{(a)}$.

If $s_{k, i}^{(b)}$ and $s_{k, i}^{(a)}$ do not contain $x$-bits then $P\left(s_{k, i}^{(a)} \mid s_{k, i}^{(b)}\right)$ is the symbol transition probability $P_{S}\left(s_{k, i}^{(a)} \mid s_{k, i}^{(b)}\right)$. Otherwise, if $s_{k, i}^{(b)}$ and $s_{k, i}^{(a)}$ contains $x$-bits, in order to evaluate $P\left(s_{k, i}^{(a)} \mid s_{k, i}^{(b)}\right)$, all possibilies of these $x$-bits need to be considered:

$$
P\left(s_{k, i}^{(a)} \mid S_{k, i}^{(b)}\right)=\frac{1}{2^{n_{k, i}^{\left(x_{i}\right)}}} \sum_{i_{2} \in S_{k, i}^{b}} \sum_{i_{1} \in S_{k, i}^{a}} P_{s}\left(i_{1}, i_{2}\right)
$$

where $S_{k, i}^{b}$ and $S_{k, i}^{a}$ is the set of all possible values of $s_{k, i}^{(b)}$ and $s_{k, i}^{(b)}$ of which $x_{u}$-bits vary in $\{0,1\}$. Thus, the number of elements of $S_{k, i}^{b}$ and $S_{k, i}^{a}$ is respectively $2^{n_{k, i}^{\left(x_{i, j}\right)}}$ and $2^{n_{k, i}^{\left(x_{u}\right)}+n_{k, i}^{\left(x_{d}\right)}}$.

Finally, the probability $P_{C}(a, b)$ is calculated by summarizing the probabilities of all possibilities.

$$
P_{C}(a, b)=\sum_{k=1}^{p_{n}} P_{p_{k}} P_{C_{k}}(a, b)
$$

where $P_{p_{k}}$ is the probability of the $k$-th allocation possibility. Normally, all the allocation possibilities can be regarded as equiprobable, i.e. $P_{p_{k}}=1 / p_{n}$.

\subsection{IA Scheme for joint source-channel coding and the given modulation type.}

The scheme is divided into 4 steps as follows:

Step 1: Training codebook with a set of training vector in order to obtain the set of codevectors $\mathbf{C}$.

Step 2: Evaluate $P_{a}(i)$ from the set of training vectors and $\mathrm{d}(i, j)$ from the codebook $\mathbf{C}$. 
Step 3: Evaluated values of parameters $\mathrm{P}_{\mathrm{C}}(i, j)$.

- Case 1: Modulation scheme is binary or QPSK with Gray mapping (BSC Channel).

- Estimate the BER $\varepsilon_{b}$.

- Calculate $\mathrm{P}_{\mathrm{C}}(i, j)$ using Eq.(6)

- Case 2: Modulation type is two-dimensional multilevel and the system model is similar as presented in Section 3.1.

- Calculate $P_{C}(i, j)$ values using method presented in Section 3.2.

- Case 3: Otherwise, values of $P_{C}(i, j)$ can be approximately estimated in the similar way described in Case 1.

Step 4: Find the optimal IA solutions using IA algorithm with input parameters $P_{a}(i), d(i, j)$ and $P_{C}(i, j)$. The codebook is then permuted by the optimized IA solution.

In the case of upgrading the existing system, this scheme will be started from step 3 .

\section{EXPERIMENT AND DISSCUSION}

\subsection{Experimental setup}

In this section, computational experiments are carried out in Matlab to examine the performance of the proposed method and to compare it with other methods.

We consider a communication system whose model is given in Section 3.1 with parameters as follows: the length of codebook $N=128$, the dimension of codevector $K=4$, the modulation is 16-QAM $(M=16, m=4)$ and 64-QAM $(M=64, m=6)$ with Gray mapping, a frame contains only one 7-bit codeword $(l=7, p=0)$. The frames are encoded by $(7,4)$ Hamming code [20] before being transmitted over the AWGN channel. Since the AWGN channel is memoryless, the concatenation of the modulator, the AWGN channel and the demodulator can be modeled as a DMC at the symbol level.

The input signals tested is 1st-order Gauss-Markov processes which were of the form:

$$
x(n)=\rho x(n-1)+w(n)
$$

where $\rho<1$ is a correlation coefficient and $w(n)$ is a zero-mean, unit variance, Gaussian white noise process. The value for $\rho$ in our experiments is 0.9.

The scheme presented in Section 3.3 is applied to this system in order to optimize the IA.

At the first step, executing LBG algorithm with splitting procedure [4] and training ratios (number of training vectors divided by $N$ ) of 1000 and squared Euclidean distance measure, codebook of 128 codevectors are generated (LBG codebook).

The input parameters of the IA problem are calculated in the next steps. The parameters $P_{C}(a, b)$ is evaluated using method described in Section 3.2 (case 2 in step 3).

A Matlab program has been written to exhaust all allocation possibilities and compute the possibility $P\left(s_{k, i}^{(a)} \mid s_{k, i}^{(b)}\right)$ for each possibility. Table 1 shows all possibilities of allocating the 7-bit codeword $b$ into symbols for the case of $M=16$.

Table 1. Possibilities of allocating the codeword $b$ into symbols (system using $(7,4)$ Hamming codes and 16 QAM modulation)

\begin{tabular}{|c|c|c|c|c|}
\hline$k$ & $s_{k, 1}^{(b)}$ & $s_{k, 2}^{(b)}$ & $s_{k, 3}^{(b)}$ & $s_{k, 4}^{(b)}$ \\
\hline 1 & $x_{d} x_{d} x_{d} b_{1}$ & $b_{2} b_{3} b_{4} x_{u}$ & $x_{u} x_{u} b_{5} b_{6}$ & $b_{7} x_{u} x_{u} x_{u}$ \\
\hline 2 & $x_{u} b_{1} x_{d} x_{d}$ & $x_{d} b_{2} b_{3} b_{4}$ & $b_{5} x_{u} x_{u} x_{u}$ & $b_{6} b_{7} x_{u} x_{u}$ \\
\hline 3 & $x_{u} x_{u} b_{1} b_{2}$ & $x_{d} x_{d} x_{d} b_{3}$ & $b_{4} b_{5} b_{6} x_{u}$ & $x_{u} x_{u} b_{7} x_{u}$ \\
\hline 4 & $x_{u} x_{u} x_{u} b_{1}$ & $b_{2} b_{3} x_{d} x_{d}$ & $x_{d} b_{4} b_{5} b_{6}$ & $b_{7} x_{u} x_{u} x_{u}$ \\
\hline 5 & $b_{1} b_{2} b_{3} b_{4}$ & $x_{u} x_{u} x_{u} b_{5}$ & $b_{6} b_{7} x_{u} x_{u}$ & \\
\hline 6 & $x_{u} x_{u} b_{1} x_{d}$ & $x_{d} x_{d} b_{2} b_{3}$ & $b_{4} b_{5} x_{u} x_{u}$ & $x_{u} b_{6} b_{7} x_{u}$ \\
\hline 7 & $x_{u} x_{u} x_{u} b_{1}$ & $b_{2} x_{d} x_{d} x_{d}$ & $b_{3} b_{4} b_{5} b_{6}$ & $x_{u} x_{u} x_{u} b_{7}$ \\
\hline 8 & $b_{1} b_{2} b_{3} x_{d}$ & $x_{d} x_{d} b_{4} b_{5}$ & $b_{6} b_{7} x_{u} x_{u}$ & \\
\hline 9 & $x_{d} b_{1} b_{2} b_{3}$ & $b_{4} x_{u} x_{u} x_{u}$ & $b_{5} b_{6} b_{7} x_{u}$ & \\
\hline 10 & $x_{u} x_{u} x_{u} b_{1}$ & $x_{d} x_{d} x_{d} b_{2}$ & $b_{3} b_{4} b_{5} x_{u}$ & $x_{u} x_{u} b_{6} b_{7}$ \\
\hline 11 & $b_{1} b_{2} x_{d} x_{d}$ & $x_{d} b_{3} b_{4} b_{5}$ & $b_{6} x_{u} x_{u} x_{u}$ & $b_{7} x_{u} x_{u} x_{u}$ \\
\hline 12 & $x_{u} b_{1} b_{2} b_{3}$ & $x_{d} x_{d} x_{d} b_{4}$ & $b_{5} b_{6} b_{7} x_{u}$ & \\
\hline 13 & $x_{d} x_{d} b_{1} b_{2}$ & $b_{3} b_{4} x_{u} x_{u}$ & $x_{u} b_{5} b_{6} b_{7}$ & \\
\hline 14 & $b_{1} x_{d} x_{d} x_{d}$ & $b_{2} b_{3} b_{4} b_{5}$ & $x_{u} x_{u} x_{u} b_{6}$ & $b_{7} x_{u} x_{u} x_{u}$ \\
\hline 15 & $x_{u} b_{1} b_{2} x_{d}$ & $x_{d} x_{d} b_{3} b_{4}$ & $b_{5} b_{6} x_{u} x_{u}$ & $x_{u} b_{7} x_{u} x_{u}$ \\
\hline 16 & $x_{d} x_{d} x_{d} b_{1}$ & $b_{2} b_{3} b_{4} x_{u}$ & $x_{u} x_{u} b_{5} b_{6}$ & $b_{7} x_{u} x_{u} x_{u}$ \\
\hline
\end{tabular}

Next, the probabilities of these allocation possibilities are computed by Eq.(8), and the codeword transition probability parameter is calculated by summarizing such probabilities by Eq.(9). 
After having determined all the input parameters, the SA algorithm [14,17] with control parameters being the same as in [17] is applied to find the suboptimal IA in the last step. The CSNR level used for IA optimization in our simulations is $5 \mathrm{~dB}$.

The resulting codebook of the proposed scheme is compared with 3 other codebook using different IA mappings: LBG IA (codebook generated by LBG algorithm with splitting procedure, mentioned in Section 2.2), IA optimized without consideration of modulation type and the random IA.

\subsection{Numerical Results and Discussion.}

The simulations were carried out to compare the performance of the entire system when using different codebooks with the same input signal source of $4,000,000$ samples corresponding to $1,000,000$ vectors. For each codebook, the signal-to-noise ratio (SNR) of the overall system defined as:

$$
\mathrm{SNR}=10 \log _{10}\left(\sigma_{\mathrm{x}} / \sigma_{\mathrm{n}}\right)[\mathrm{dB}]
$$

where $\sigma_{x}$ and $\sigma_{n}$ are the signal and noise variances respectively, is calculated with the channel SNR (CSNR) ranging from $0 \div 25 \mathrm{~dB}$.

Fig. 5 shows the SNR of the overall system for each method against the CSNR. It can be seen that the IA optimized by the method with consideration to the given modulation type provides the best performance in comparison to others in terms of higher SNR. Also, it is seen that the systems using the optimized IA mappings outperform the systems with no IA optimization (the LBG IA and the random IA).

When the CSNR is high enough, the channel can be assumed to be error-free and the SNR of the entire system with different codebooks reach the same highest level, also known as the Signal-toQuantization Noise Ratio $\left(\mathrm{SN}_{\mathrm{q}} \mathrm{R}\right)$. The reason is that these four codebooks are basically the same, but using different IA mappings.

At low CSNR levels $(0 \div 5 \mathrm{~dB})$, the performance of the system using the proposed IA scheme is not better than using other optimized IA schemes. The more accurate parameter estimation in this case is no longer an advantage. This is because there will be a large number of errors in the parity bits as well as in the bits of data, so the FEC mechanism may cause more errors when decoding with incorrect parity bits. However, under such a bad condition, the optimized IA mappings still have better performance than the random IA mapping. It can be observed that when the FEC fails, optimal IA can be a last line of defence against channel errors.

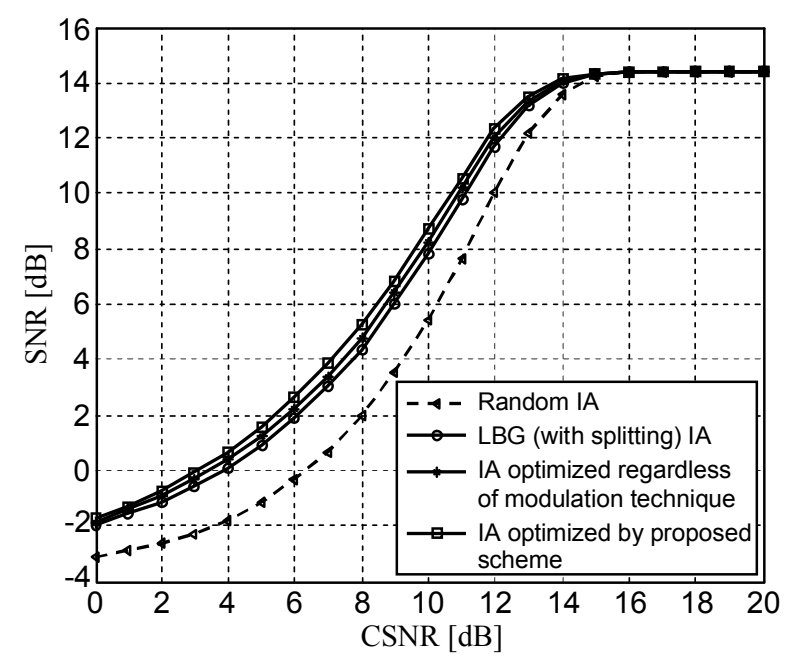

(a) $M=16, m=4(16 \mathrm{QAM})$

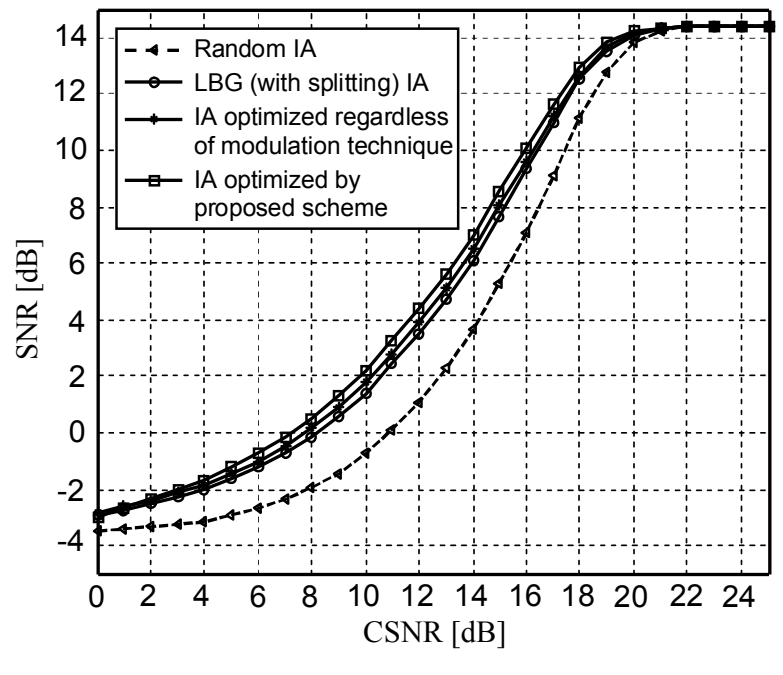

(b) $M=64, m=6$ (64 QAM)

Fig.5. Performance comparison of system using various codebooks

Through the above experiments, the effectiveness of the proposed IA scheme is verified. Using the proposed method can be improve the error resilience of the system without sacrificing the transmission rate and the system complexity. 


\section{CONCLUSION}

In this paper, we investigated the Index Assignment problem with consideration to the given modulation type and propose an IA scheme for joint source channel coding and various digital modulation types. The proposed scheme can be combined with other error control or mitigation methods to more improve the error resilience of the communication system. This study also considers the combination between the IA scheme and FEC coding, and the performance of the proposed scheme is evaluated by simulations.

Theoretical analysis and simulations have shown that systems using IA optimization scheme with consideration to the given modulation type provide better performance than systems using IA optimization regardless of the modulation type. The simulations also demonstrated the important role of IA optimization by the significant difference between the overall SNR of the system using optimized IA schemes and the random scheme. However, the proposed scheme required more complexity in design but this is not a serious problem since optimal codebook design is a one-time process.

The disadvantage of this JSCC scheme is that the optimized coder is integrated with the specific source and channel therefore it needs to be re-optimization when changing source or channel. When combining with FEC coding, the proposed scheme can be associated with systematic codes only.

Studies are in process to optimize more complex coding structures, such as MultiStage VQ, Switched Split VQ, Switched MultiStage VQ. In addition, combining source channel coding and modulation scheme in more general cases with other channel models and its application for specific communication systems is ongoing.

\section{REFERENCES}

[1] C.E. Shannon, "A Mathematical Theory of Communication", Bell System Techical Journal, vol. 27, pp. 379-423, 623-656, 1948.

[2] A. Gersbo and R. Gray, "Vector quantization and signal compression", Boston, Ma. Kluwer Academic Publishers, 1992.
[3] L. Xiao and X. Dong, "The exact transition probability and bit error probability of twodimensional signaling", IEEE Trans. Wireless Commun., vol.4, pp. 2600-2609, Sep. 2005.

[4] Y. Linde, A. Buzo, and R. M. Gray, "An algorithm for vector quantization design", IEEE Trans. on Commun., Vol. COM-28, pp. 84-95, 1980.

[5] S. B. Z. Azami, P. Duhamel, and O. Rioul, "Joint source-channel coding: Panorama of methods," Proceedings of CNESWorkshop on Data Compression, pp 1232-1254, 1996.

[6] Tzu-Chuen Lu, and Ching-Yun Chang. "A survey of VQ codebook generation", Journal of Inf. Hiding and Multimedia Signal Processing, Ubiquitous International, Vol. 1, No. 3, pp. 190-203, 2010.

[7] H. Skinnemoen, "Combined Source-Channel Coding with Modulation Organized Vector Quantization (MOR-VQ)", Proc. IEEE GLOBECOM, vol. 2. IEEE, Nov. 1994, pp. 853-857.

[8] T.N. Tuan, N.Q. Trung, "Index Assignment Optimization for Digital Communication Systems using M-ary Modulation Schemes", Special Issues on Research and Development on Inf. \& Commun. Tech., Vietnamese Journal on Information Communications Technology, vol E-3, No. 8(12), pp. 38-47, 2015.

[9] K. Ramchandran et al., "Multiresolution broadcast for digital HDTV using joint source/channel coding", IEEE Journal on Selected Areas in Commun., vol. 11.1, pp. 6-23, Jan. 1993.

[10] A. Fuldseth and J. M. Lervik, "Combined source and channel coding for channels with a power constraint and multilevel signaling", Proc. ITG Conference, (Munchen, Germany), pp. 429-436, ITG, Oct. 1994.

[11] J.-K. Han and H.-M. Kim, "Joint optimization of VQ codebooks and QAM signal constellations for AWGN channels", IEEE Trans. on Commun., Vol. 49, Iss. 5, pp 816-825, 2001.

[12] H. Elmeddeb, et al, "Combined Adaptive Modulation and Channel Optimized Vector Quantization", IEEEEURASIP-ISCCSP, 2006.

[13] K. Zeger and A. Gersho, "Pseudo-Gray Coding", IEEE Trans. on Commun., Vol. 38, No. 12, 2147-2158, 1990.

[14] N. Farvadin, "A Study of Vector Quantization for Noisy Channels", IEEE Trans. on Inf. Theory, Vol. 36, No. 4, 799-809, 1990.

[15] A. Miri, E. Hons and A.K. Khandani, "Optimising the combined source and channel coding of a discrete communication system". IEE Proc. Commun., vol. 152 , iss. 3, pp. 282-287, 2005.

[16] W. Yue, "An Index Assignment Algorithm over Noisy Channel", Software Engineering and Knowledge 
Engineering: Theory and Practice, Springer Berlin Heidelberg, 2012, pp. 663-671.

[17] T.N. Tuan, N.Q. Trung, "Improving the Simulated Annealing algorithm for the Index Assignment method to enhance the robustness of communication systems", Special Issues on Research, Dev. and Appl. on Inf. \& Commun. Tech., Vietnamese Journal on Inf. Tech. \& Commun., vol E-3, No. 7(11), pp. 13-20, 2014.

[18] H. Oztoprak, S. Villette and A. Kondoz, "Index assignment-based channel coding", IET Commun., vol. 6, iss. 2, pp. 172-178, 2012.

[19] Xiaolin Wu, et al, "On computation of performance bounds of optimal index assignment", IEEE Trans. on Commun., vol. 59, pp. 3229-3233, 2011.

[20] C.G. Clark Jr., J.B. Cain, "Error-correction coding for digital communications", Springer Science \& Business Media, 2013.

\section{AUTHORS' BIOGRAPHIES}

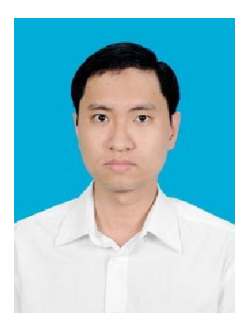

Tran Ngoc Tuan is working at School of Electronics and Telecommunications, Hanoi University of Science and Technology (HUST), Vietnam. He received the Engineer and M.Sc. degrees in electrical engineering from HUST in 2005 and 2008. Currently he is a $\mathrm{PhD}$ candidate at School of Electronics and Telecommunication, HUST. His research interests include digital signal processing, speech coding and joint source channel coding.

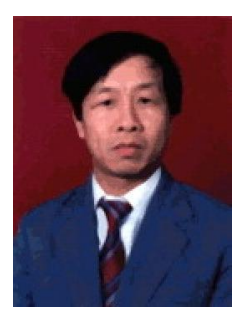

Nguyen Quoc Trung is working at School of Electronics and Telecommunications, Hanoi University of Science and Technology, Vietnam. He received Ph.D degree from Hungarian Academy of Science in 1982. He was promoted to Accociate Professor in 2004. His research interests include digital signal processing, subband coding and digital communication. 